\title{
ZYGAENIDAE (LEPIDOPTERA) IN THE LEPIDOPTERA COLLECTIONS OF THE CROATIAN NATURAL HISTORY MUSEUM
}

\author{
Martina ŠAšić ${ }^{1}$ Ana NAhirnić ${ }^{2}$ \& Gerhard M. TARMANN ${ }^{3}$ \\ ${ }^{1}$ Department of Zoology, Croatian Natural History Museum, Demetrova 1, HR-10000 Zagreb, Croatia \\ ${ }^{2}$ National Museum of Natural History, Tsar Osvoboditel Blvd 1, BG-1000 Sofia, Bulgaria \\ ${ }^{3}$ Tiroler Landesmuseen, Ferdinandeum, Naturwissenschaftliche Sammlungen, Feldstrasse 11a, \\ A-6020 Innsbruck, Austria
}

Šašić, M., Nahirnić, A. \& Tarmann, G. M.: Zygaenidae (Lepidoptera) in the Lepidoptera collections of the Croatian Natural History Museum. Nat. Croat., Vol. 25, No. 2., 233-248, 2016, Zagreb.

We examined 581 specimens from the family Zygaenidae (Lepidoptera) from the Balkans and the surrounding area (Croatia, Macedonia, Serbia, Slovenia) in the collections of Croatian Natural History Museum in Zagreb. Almost all species occurring in Croatia are represented in the collections of the Croatian Natural History Museum. Despite the small number of specimens stored in this museum Zygaenidae of Croatia are well studied comparing with other countries of the Balkan Peninsula.

Key words: Lepidoptera, Zygaenidae, Balkan Peninsula, Croatian Natural History Museum, collection

Šašić, M., Nahirnić, A. \& Tarmann, G. M.: Zygaenidae (Lepidoptera) u zbirkama leptira Hrvatskoga prirodoslovnoga muzeja. Nat. Croat., Vol. 25, No. 2., 233-248, 2016, Zagreb.

Pregledan je 581 primjerak leptira iz porodice Zygaenidae iz zbirki leptira Hrvatskoga prirodoslovnoga muzeja sa šireg područja Balkana (Hrvatska, Makedonija, Srbija, Slovenija). U zbirci su pohranjene sve vrste koje su zastupljene $u$ fauni te porodice u Hrvatskoj. Unatoč relativno malom broju primjeraka u zbirkama, porodica Zygaenidae u Hrvatskoj je dobro istražena u usporedbi s drugim dijelovima Balkanskog poluotoka. $\mathrm{ka}$

Ključne riječi: Lepidoptera, Zygaenidae, Balkanski poluotok, Hrvatski prirodoslovni muzej, zbir-

\section{INTRODUCTION}

The Croatian Natural History Museum was founded in 1846 and from that time it collects, purchases, cares and studies natural treasures from Croatia and neighbouring countries. Today it houses about 2 million specimens systematized in more than 100 collections.

The Lepidoptera collection has about 90000 specimens organized in ten collections. Burnets and forester moths (Zygaenidae) have never been systematically collected because of limited interest to this family and possibly because of their known resistance to cyanide, which was a standard method for collecting butterflies and moths. Therefore, only a limited number of specimens are held in our collection. A part of this collection has already been reported in Kučinić et al. (1994), Mladinov (1958, 1965, 1967, 1976, 1978). 
Faunistic papers on Lepidoptera of Croatia which include Zygaenidae have been published throughout the two previous centuries. Early data are e.g. included in AbafiAigner et al. (1896, 1910), Bohatsch (1892), Carrara (1846), Duponchel (1844 [1846]), HAfNer (1994), Lederer (1853), MANn (1857, 1867, 1869), Schwingenschuss \& Wagner (1925), StAuder (1920; 1921), Vukotinović (1879) while recent results come mainly from anecdotal observations (Kranjčev, 1985; Kučinić, 1998; Mladinov, 1965, 1978; Habeler 2003). Only a few papers deal entirely with this group in Croatia (STAUdER, 1921, 19301932, RAUCH 1981).

Natural history collections act as repositories and play a vital role in understanding biodiversity. As habitat loss is one of the greatest threats to biodiversity today and collections provide data on these changes our aim was to collect all the available data on this family group in Croatia as a part of "Zygaenidae of the Balkan Peninsula“ project in order to facilitate future work on this insect group.

Zygaenidae are extremely stenoecious insects and are therefore good indicator species for environmental changes. They can show us perfectly whether a biotope is in a good 'natural' condition or somehow disturbed or even contaminated (TARMANN, 2004). Special studies on this topic have been undertaken in northern Italy in Val Venosta (Vinschgau) (TARMANN 2000a, 2000b, 2009) where it has been shown that species of the genera Adscita Retzius, 1783, Jordanita Verity, 1946, Rhagades Wallengren, 1863, and Zygaena Fabricius, 1775, are excellent indicators of pesticide use in the intensive apple cultures. It has been shown that the poison was transported by wind into the environment but not only to the adjacent areas but also into biotopes that are kilometres away. Zygaenidae were amongst the first insects that disappeared from seemingly completely intact environment. Several of such biotopes were even Natura 2000 sites under European protection. Zygaenidae are day active and easy to observe. Together with butterflies they have proved to be perfect indicators for monitoring destructive events caused by moulting inhibitors (e.g. Dimilin, Insegar etc.) that are used against the codling moths (Cydia pomonella (Linnaeus, 1758)) (Tortricidae) and similar species in fruit plantations (TARMANN 2000a, 2000b). Today, in a time of intense anthropogenic impacts on ecosystems, such data allow us to understand the changes around us better, which are manifested as changes in the composition of biological diversity of an area regardless of whether they are the result of long-term research or just based on individual species.

\section{MATERIAL AND METHODS}

We examined 581 specimens from the Balkans and the surrounding area (Croatia, Macedonia, Serbia, Slovenia) in the collections of the Croatian Natural History Museum in Zagreb. The specimens are deposited in two main collections: Central Lepidoptera collection and Collection Lorković. The number of specimens in the Central Lepidoptera Collection is 843 and together with the specimens from Collection Lorković and additional material from the fieldwork it amounts to around 1000. As the Central Lepidoptera collection originates from several older donations (ex coll. Gušić, ex coll. Koča, ex coll. Kozulić, ex coll. Locke, ex coll Grund) we provide in brackets the abbreviations of these collections and in recent material collected by museum curators the collectors are mentioned. In special cases, e.g. of father and son Igalffy, where the first name is not mentioned on the label as collector we write only Igalffy. Specimens in the Central Collection which were collected and put directly into the collection have the name of the legator and are marked as 'coll. Central'. The Lorković collection was collected by the famous 


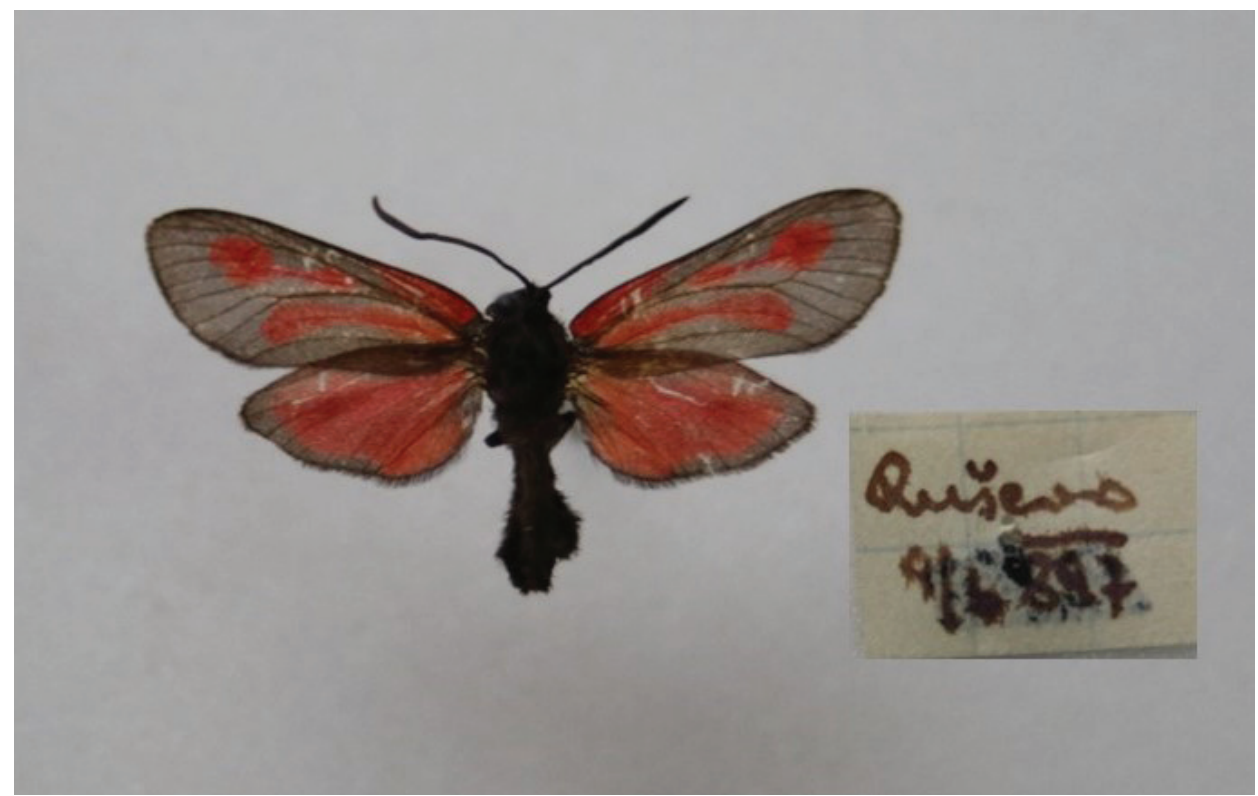

Fig. 1. Zygaena osterodensis Reiss, 1921, specimen from ex coll. Koča, CNHM.

Croatian lepidopterologist Zdravko Lorković and is abbreviated as 'coll. Lorković' as well as the material collected by Dragan Rucner is abbreviated as 'coll. Rucner'.

In the case where museum material could be connected with the published material the reference of the publication is given in brackets. The identification of species is based on habitus and genitalia examination. Genitalia slide numbers are placed in square brackets and were done mainly by Lidija Mladinov and by the second author. When genitalia of Z. purpuralis were not placed in Euparal on slide they were placed in small tubes filled with glycerol. The systematics follows EfETov \& TARMAnN (1999) and HofMANN \& TREMEWAN (1996).

\section{RESULTS AND DISCUSSION}

The analysed collections of Zygaenidae comprise 24 species (with 581 specimens) of which 10 species with 97 specimens belong to the subfamily Procridinae and 14 species with 484 specimens to Zygaeninae. The majority of the specimens of the Z. purpuralis complex from the collection are included in this list but examination of genitalia of some specimens, essential for a proper determination, had to be postponed.

The examined species originate from the following six countries: Bosnia and Herzegovina 4 (15 specimens), Croatia 22 (452), Macedonia 3 (4), Montenegro 5 (29), Serbia 2 (3) and Slovenia 9 (78). J. budensis published in Mladinov (1958) collected by Valjavec on 12.07.1923 is not deposited in the collection and could not be verified.

J. budensis published by KočA (1901) from Križpolje is J. globulariae (see below) but the second record from Brinje could not be traced. As this second specimen was collected at the same date we doubt that it is J. budensis. 


\section{List of the species:}

Family ZYGAENIDAE Latreille, 1809

Subfamily Procridinae Boisduval, 1828

1. Theresimima ampellophaga (Bayle-Barelle, 1808)

HR: Vinkovci 19.06.1908. ex coll. Koča $1 \mathrm{~m}$

2. Rhagades pruni ([Denis \& Schiffermüller], 1775)

Rhagades pruni pruni ([Denis \& Schiffermüller], 1775)

HR: Bosiljevo ex coll. Kozulić $1 \mathrm{~m}$

Klek 03.08.1916. ex coll Gušić $1 \mathrm{~m}$

Vinkovci 12.07.1908. ex coll. Koča 1m

\section{Adscita statices (Linnaeus, 1758)}

Adscita statices statices (Linnaeus, 1758)

HR: Bjelolasica (Velika Kapela) 1500 m 23.07.1954. leg. Z. Lorković, coll. Lorković 1 m Boljara (=Boljare) (Žumberak Mt.), VL 26, 4.07.1995. leg. M. Vajdić

Brod na Savi (= Slavonski Brod) 21.06.1909. ex coll. Koča $1 \mathrm{~m}$

Hrvatsko 08-10.06.1973. 1 m, 16.06.1974. leg. L. Mladinov coll. Central 1 m (MLAdinov, 1976)

Hrvatsko-Kupa 29.06.1976. 1 m, 1 f, 14.06.1981. 1 m, both leg. L. Mladinov

Krk Jezero 17.06.1998. $1 \mathrm{~m} 1 \mathrm{f}$

Leska (Risnjak Mt.) 24.06.1994. leg. M. Kučinić $1 \mathrm{~m} 1 \mathrm{f}$

Mala Kapela 06.1960. leg. I. Igalffy $1 \mathrm{~m}, 1 \mathrm{f}$

Maksimir (Zagreb) 02.07.1931. ex coll. Valjavec $1 \mathrm{~m}$

Mrkopalj 10.07.1918. ex coll. Gušić det. L. Mladinov $1 \mathrm{~m}$ [603]

Plitvički Ljeskovac 27.07.1962. leg. Z. Lorković coll. Lorković $1 \mathrm{~m} 1 \mathrm{f}$

Trnovec 07. 1929. $1 \mathrm{~m}, 1$ f, 06.1914. $1 \mathrm{~m}$, all leg. I. Igalffy, coll. Central

Tuškanac (Zagreb) 27.06.1922. leg. Z. Lorković coll. Lorković $1 \mathrm{~m}$

Vinkovci 26.06.1909. ex coll. Koča $1 \mathrm{~m}$

SLO: Osilnica 29.06.1971. $1 \mathrm{~m}$ [533] (Mladinov, 1976)

Osilnica 21-28.06.1970. 2 m 2 f [448, 449], 11.06.1972. 2 m 1 f [575], all leg. L. Mladinov (Mladinov, 1976)

Podvrh 13.06.1972. leg. L. Mladinov $2 \mathrm{~m} 4 \mathrm{f}$ [578] (Mladinov, 1976)

Prebold 23.07.1965. leg. L. Mladinov gen. det. L. Mladinov $1 \mathrm{~m}$

\section{Adscita geryon (Hübner, 1813)}

Adscita geryon orientalis (Alberti, 1938)

HR: Bosiljevo ex. coll. Kozulić det. Mladinov 1 m [596]

Adscita geryon was mentioned by Rebel (1910) for 'Dalmatien' and by Stauder (1921) for 'Küstenland Istrien' and 'Dalmatien' without exact locality. So far, no specimens could be found by us in collections from Croatia except that mentioned in this paper. Adscita geryon is surprisingly rare in Croatia. The only confirmed record (by genitalia dissection) from a locality near the Slovenian border does not seem to match the true distribution of this species in Croatia. A. geryon orientalis was described from the Bosnian Mountains (Makljen pass) but must occur in more parts of the Dinaric Alps. However, this has to be confirmed in future. 


\section{Adscita mannii (Lederer, 1853)}

HR: Boljare (Žumberak) 28.06.1994. leg. M. Šašić 2 m

Brseč 06.06.1970. leg. K. Igalffy $4 \mathrm{~m}$

Braslovje - Rude 500 m 07.06.1966. leg. Z. Lorković coll. Lorković $1 \mathrm{~m}$

Čepić polje (Istria), VL03, 16.06.1999. leg. M. Šašić 1 f

Đurđevački pijesci 29.06.1999. leg. M. Šašić $1 \mathrm{f}$

Đurđevački pijesci 30.06.1999. leg. M. Šašić $1 \mathrm{~m}$

Korita (NE of Knin) 31.05.2014. leg. N. Tvrtković 1m

Kuna (Sniježnica Mt.) 20.05.2000. leg. M. Vajdić 1 f [968]

Sveta Gera (Žumberak Mt.) 19.06.1917. ex. coll. Gušić 2 m [605]

Sveta Gera, Izvor (Žumberak Mt.) 23.07.1991. leg. M. Vajdić 2 m

\section{Jordanita notata (Zeller, 1847)}

HR: Divoselo - Klisa (Gospić) 09.06.1973. leg. Z. Lorković coll. Lorković 2 m

BIH: Gusni put (Maglić Mt.) 02.07.1926. ex. coll. Gušić 5 m

Gusni put (Maglić Mt.) 06.07.1926. ex. coll. Gušić 1 m [600]

Sutjeska - Maglić 01.07.1926. ex. coll. Gušić $1 \mathrm{~m}$

\section{Jordanita graeca (Jordan, 1907)}

Jordanita graeca graeca (Jordan, 1907)

HR: Zara (= Zadar) 1887 ex coll. Locke $1 \mathrm{~m}$

MK: Ohrid 15.06.1961. leg. L. Mladinov 1 m 1 f [606]

\section{Jordanita chloros (Hübner, 1813)}

Jordanita chloros chloros (Hübner, 1813)

HR: Brseč, Golovik 06.1973. leg. I. Igalffy $1 \mathrm{~m}$

\section{Jordanita globulariae (Hübner, 1793)}

HR: Bosiljevo ex. coll. Kozulić det L. Mladinov 1 m (MLAdinov, 1976)

Caska (Pag) 02.07.1962. det. L. Madinov 1 m [226] (Mladinov, 1965)

Delnice 15.07.1916. ex. coll. Gušić $1 \mathrm{~m}$

Hrvatsko 02.07.1971. det. L. Mladinov 1 m (Mladinov, 1976)

Hrvatsko-Kupa 21.06.1975. leg. L. Mladinov 2 m (Mladinov, 1976)

Hrvatsko-Kupa 10.06.1979. leg. Mladinov $1 \mathrm{~m}$

Kapela (Lička Plješevica) 05.07.1975. leg. K. Igalffy $1 \mathrm{~m}$

Klek 03.07.1916. ex coll. Gušić, det. L. Mladinov $1 \mathrm{~m}$

Križpolje 07.07.1894. ex. coll. Koča 1 m (published as Ino budensis in KočA (1901)

Križpolje 07.07.1916. ex. coll. Gušić det. L. Mladinov $1 \mathrm{~m}$

Maksimir (Zagreb) 07.1924. ex. coll. Gušić det. L. Mladinov 1m (Mladinov, 1958)

Omišalj 13.08.1976. leg. L. Mladinov $1 \mathrm{~m}$

Plitvički Ljeskovac 23.07.1962. leg. Z. Lorković coll. Lorković 1 m 1 f

Vinkovci 03.07.1900. $1 \mathrm{f}$ [607]

Vinkovci 14.08.1909. $1 \mathrm{~m}, 04.07 .1910 .1 \mathrm{f}$ both ex coll. Gušić

Zagreb 06.07.1976. coll. Rucner $1 \mathrm{~m}$

Zelengaj (Zagreb) 14.07.1918. coll. Lorković $1 \mathrm{f}$

SLO: Podvrh 13.06.1972. leg. L. Mladinov 4 m 1 f [576, 577] (Mladinov, 1976) 


\section{Jordanita subsolana (Staudinger, 1862)}

HR: Caska (Pag) 29.06.1962 leg. L. Mladinov 1 m [227] (Mladinov, 1965)

Sveta Gera 19.06.1917. ex. coll. Gušić 1 f

Subfamily Zygaeninae Latreille, 1809

\section{Zygaena punctum Ochsenheimer, 1808}

Zygaena punctum dalmatina Boisduval, 1834

HR: Brseč, Golovik 06.1973. leg. K. Igalffy $1 \mathrm{~m}$

Caska (Pag) 10.06.1958. $1 \mathrm{~m}$ [85], 21.06.1960. 2 m, 22.06.1960. 1 m, 29.06.1962. 2 f, all leg.

L. Mladinov (Mladinov, 1965)

Dubrava (Pag) 09.07.1962. leg. K. Igalffy coll. Central $1 \mathrm{~m}$

Dubrovnik 06.1950. leg. K. Igalffy coll. Central $2 \mathrm{~m}$

Kolansko Blato (Pag) 11.06.1958. leg. L. Mladinov coll. Central 1 m [86] (Mladinov, 1965)

Pag 07.1960. leg. Igalffy coll Central $2 \mathrm{~m}$

Rab 08.07.1962. leg. K. Igalffy $1 \mathrm{~m}$

Senj 19.06.1973. leg. L. Mladinov $1 \mathrm{~m} 1 \mathrm{f}$

Susak 06.1962. $1 \mathrm{~m}, 13.06 .1962 .2 \mathrm{~m}$ all leg. L. Mladinov

Unije 25.06.1963. leg. L. Mladinov coll. Central $1 \mathrm{~m}$ (Mladinov, 1967)

Vozilići 27.06.1974. $1 \mathrm{~m} 1$ f, 20.06.1975. $1 \mathrm{~m} 1$ f, 29.06.1978. 1 f, all leg. F. Perović

MNE: Herceg Novi 06.1954. leg. Igalffy coll. Central $1 \mathrm{~m}$

\section{Zygena cynarae (Esper, 1789)}

Zygaena cynarae cynarae Esper, 1789

HR: Gornje Čučerje (Zagreb) 01.07.1990. leg. Z. Lorković coll. Lorković 1 m

Zygaena cynarae adriatica Burgeff, 1926

HR: Bosiljevo ex coll. Kozulić $1 \mathrm{~m}$

Caska (Pag) 25.06.1962. leg. L. Mladinov $1 \mathrm{~m}$

Krk Jezero 17.06.1998. leg. M. Kučinić $1 \mathrm{~m}$

Zadar 18871 m, 18901 m, both ex coll. Locke

\section{Zygaena brizae (Esper, 1800)}

Zygaena brizae brizae (Esper, 1800)

SRB: Sonta 16-17.06.1985. leg. Z. Lorković coll. Lorković 2 m

This record is significant since Z. brizae is very rare in Serbia (JAKšić \& Ristić, 1999; NAHiRnić et al., 2012).

\section{Zygaena purpuralis (Brünnich, 1763)}

Z. purpuralis purpuralis Brünnich, 1763

HR: Bosiljevo, ex. coll. Kozulić 1 m 1 f [969]

In the region Z. purpuralis purpuralis occurs in Pannonian Basin. This is the only known record of the nominotypical subspecies in Croatia. As there are no records for the continental Croatia except for Zagreb area we suppose that this subspecies occurs in Slavonia with the contact zone with Z. purpuralis lathyri in Central Croatia. 


\section{Zygaena purpuralis lathyri Boisduval, 1828}

HR: Begovo Razdolje 04.08.1955. leg. Z. Lorković coll. Lorković $1 \mathrm{~m}$

Boljara (=Boljare) (Mt. Žumberak) 23.06.1994. leg. Z. Lorković coll. Lorković 1 m, 04.07.1995. leg. M. Vajdić $1 \mathrm{~m}$

Dinara Mt. 28.06.1929. ex. coll. Valjavec $1 \mathrm{~m}$ [842]

Hrvatsko 22.06.1975. leg. L. Mladinov coll. Central $1 \mathrm{~m}$

Kapela (Mt. Lička Plješevica) 05.07.1979. leg. Igalffy 1 m [970]

Lipovečka Gradna (Samoborsko Gorje) 22.06.1954. leg. Z. Lorković coll. Lorković 1 m, $1 \mathrm{f}[971]$

Ljubina poljana (Mt. Poštak) 07.06.2014. leg. N. Tvrtković $1 \mathrm{~m}$

Mala Kapela Mt. 06.1960. coll. Central $1 \mathrm{~m}$

Palačnik (Samoborsko Gorje) 19.06.1955. leg. Z. Lorković coll. Lorković 2 m [972]

Plitvički Ljeskovac 07.1962. leg. Z. Lorković coll. Lorković $1 \mathrm{~m}$

Samoborsko Gorje 10.07.1991. leg. Z. Lorković coll. Lorković $1 \mathrm{~m}$

Zagreb, Jurjevska ulica/ street 03.08.1919. leg. Z. Lorković coll. Lorković $1 \mathrm{~m}$

Zelengaj (Zagreb) 07.07.1918. leg. Z. Lorković coll. Lorković $1 \mathrm{~m}$

MK: Ceripašina planina (Šar-planina Mts) 2000-2200 m 26.07.1952. leg. Z. Lorković

coll. Lorković $1 \mathrm{~m}$

MNE: Durmitor Mt. 24.07.1926. ex. coll. Gušić 1 m [841]

SLO: Ložec 21.06.1975. leg. L. Mladinov coll. Central $1 \mathrm{~m}$

Osilnica 21-28.06.1970. leg. gen. det. L. Mladinov coll. Central 1 m [450] (MLAdinov, 1976)

Osilnica 02.07.1971. coll. Central 2 m [523] (Mladinov, 1976)

Osilnica 21-28.06.1970. 2 m [973], 15.07.1974. $1 \mathrm{~m}$ all leg. L. Mladinov coll. Central

Podvrh 13.06.1972. $1 \mathrm{~m}$ [581] (Mladinov, 1976), $1 \mathrm{~m}$ all coll. Central

Srobotnik 14.06.1981 leg. L. Mladinov coll. Central $1 \mathrm{f}$

This subspecies occurs mainly in the mountain regions of the Dinaric Alps. Specimens are robust, hairy and dark, with broad hindwing border, males tend to have reduced distal end of streak (3+5). NAUMANN et al. (1983) published the only reliable records on the presence of Zygaena purpuralis in Croatia, all based on genitalia morphology. They conclude that Zygaena purpuralis is the only species of the purpuralis-minos-diaphanagroup present in the northern and central Balkans.

As Z. minos ([Denis \& Schiffermüller], 1775) is present in neighbouring countries Hungary, Bosnia \& Herzegovina (NAumann et al., 1983) and Montenegro (NAHIRnić \& TARMANN, 2013), its presence is also expected in Croatia, even so very local.

\section{Zygaena carniolica (Scopoli, 1763)}

Zygaena carniolica flaveola Esper, 1786

HR: Trnjani 1908. ex coll. Gušić. $1 \mathrm{~m}$

Vinkovci 1909. ex coll. Gušić 1 f

\section{Zygaena carniolica carniolica (Scopoli, 1763)}

HR: Bela - Podrute (Ivanščica Mt.) 05.07.1964. leg. Z. Lorković coll. Lorković $1 \mathrm{f}$

Bosiljevo ex coll. Kozulić 1 f (Mladinov, 1976)

Bosiljevo ex coll. Gušić $1 \mathrm{f}$

Braslovje (Rude) 500 m 07.06.1966. leg. Z. Lorković coll. Lorković 1 m 
Caska (Pag) 09.06.1958. 1 m 2f, 24.06.1960. 2 m, 08.07.1960. 1 m, 25.06.1962. leg. L. Mladinov coll. Central $2 \mathrm{~m} 1 \mathrm{f}$ (Mladinov, 1965)

Košljun (Pag) 02.07.1956. coll. Central 1 f (Mladinov, 1965)

Kraljevec 22.07.1924. ex. coll. Gušić 1 m (MLAdinov, 1958)

Krpelj 13.07.1916. ex. coll. Gušić $1 \mathrm{~m}$

Lađana (Biokovo) 23.07.1995. leg. M. Vajdić $1 \mathrm{f}$

Male Srakane 18.05.1962. leg. A. Magerle coll. Central $1 \mathrm{~m}$

Mali Lošinj 20.05.1974. 1 m, 21.05.1974. 1 f, 23.05.1974. 1 m, all leg. K. Igalffy

Ogulin 12.07.1916. ex. coll. Gušić $1 \mathrm{~m}$

Poklek (Žumberak Mt.) 22.07.1991. leg. M. Vajdić $1 \mathrm{f}$

Plitvički Ljeskovac 23.07.1982. leg. Z. Lorković coll. Lorković 1 m; 25.07.1998. leg. M.

Vajdić $2 \mathrm{~m}, 2 \mathrm{f}$

Plitvički Crni vrh 26.07.1961. leg. Z. Lorković coll. Lorković $1 \mathrm{~m} 1 \mathrm{f}$

Povljana (Pag) 26.06.1962. leg. K. Igalffy coll. Central 1 m (MLAdinov, 1965)

Rab 08.07.1962. leg. K. Igalffy $1 \mathrm{~m}$

Rab (Rab) 05.07.1964. leg. Z. Lorković coll. Lorković $1 \mathrm{~m} 1 \mathrm{f}$

Sljeme 28.07.1923. ex. coll. Gušić 1 m (Mladinov, 1958)

Strahinjščica 22.07.1917. ex. coll. Gušić $1 \mathrm{~m}$

Susak 22.06.1962. leg A. Magerle coll Central $1 \mathrm{~m}$

Šestine 29.07.1923. ex. coll. Valjavec $2 \mathrm{f}$

Trnovec 07.1929. 1 m 1 f, 03.07.1971. 1 m (MLadinov, 1976), 16.07.1981. 1 f all leg. K. Igalffy coll. Central

Unije 25.06.1963. leg. L. Mladinov coll. Central 2 m 2 f (Mladinov, 1967)

Vozilići 20.06.1975. leg. F. Perović $4 \mathrm{~m} 1 \mathrm{f}$

Zadar 1890 ex. coll. Locke Wien $2 \mathrm{f}$

Zagreb 12.07.1976. 1 f, 15.07.1976. $1 \mathrm{~m}$ all coll. Rucner

MNE: Lovćen 27.07.1926. ex. coll. Gušić $1 \mathrm{f}$

SLO: Čačići 26.07.1975. leg. L. Mladinov 1 m (Mladinov, 1976)

16. Zygaena loti ([Denis \& Schiffermüller], 1775)

Zygaena loti balcanica Reiss, 1922

BIH: Strmica 10.06.1929. ex. coll. Valjavec $1 \mathrm{~m}$

Sustavac (Treskavica Mt.) 04.07.1968. leg. K. Igalffy coll. Central 3 m

HR: Kapela (Lička Plješevica) 08.08.1980. leg. K. Igalffy coll. Central 2 f

Klek 09.06.1916. ex coll. Gušić $1 \mathrm{~m}$

Mala Bubinka (Lička Plješevica) 08.08.1974. leg. Igalffy $1 \mathrm{~m}$

Zygaena loti praeclara Burgeff, 1926

HR: Bračana, VL 22, 10.05.2001. leg. M. Šašić $1 \mathrm{~m}$

Brseč 05.06.1970. leg. K. Igalffy $5 \mathrm{~m} 1 \mathrm{f}$

Brseč, Golovik 06.1973. leg. K. Igalffy $1 \mathrm{~m} 1 \mathrm{f}$

Caska (Pag)25.06.1961. leg. L. Mladinov coll. Central 2 m 1 f [259] (MLAdinov, 1965)

Caska (Pag) 29.06.1961. leg. L. Mladinov coll. Central 1 m 3 f [260, 258] (Mladinov, 1965)

Knin 16.06.1926. ex. coll. Valjavec $1 \mathrm{f}$ 
Ložec 11.06.1977. leg. L. Mladinov 1 f (MLAdinov, 1978), 13.07.1980. leg. L. Mladinov coll. Central $1 \mathrm{~m}$

Lošinj 05.1907. 1 f, 07.1908. leg. Igalffy coll. Central $1 \mathrm{~m}$

Marušići (Istra) 17.05.2000. leg. M. Šašić $1 \mathrm{~m}$

Novi Vinodolski 15.06.1973. leg. Z. Lorković coll. Lorković $1 \mathrm{~m}$

Rab 08.07.1962. leg. K. Igalffy $1 \mathrm{f}$

Rab 09.06.1921. leg. Z. Lorković coll. Lorković $1 \mathrm{~m}$

Vozilići 09.06.1976. 1 m, 23.05.1975. 1 f both leg. F. Perović

Vratnik 16.07.1978. leg. L. Mladinov coll. Central $1 \mathrm{~m}$

\section{Zygaena loti loti ([Denis \& Schiffermüller], 1775)}

HR: Lipovečka Gradina (Samoborsko gorje) 22.06.1954. leg. Z. Lorković coll. Lorković $1 \mathrm{~m}$

Palačnik (Zagreb) 19.06.1955. leg. Z. Lorković coll. Lorković 2 m (MLADinov, 1958)

Pustodol (Medvednica) 12.06.1980. leg. Z. Lorković coll. Lorković $1 \mathrm{~m}$

Strahinščica 29.06.1902. ex. coll. Gušić $1 \mathrm{~m}$

Šušnjevec (Zagreb) 08.06.1966. leg. Z. Lorković coll. Lorković 2 m

Vinkovci 15.07.1908. 1 m, 05.07.1908. 1 f both ex. coll. Gušić

SLO: Fala na Dravi 10.07.1932. leg. Lorković coll. Lorković $1 \mathrm{~m}$

Fala na Dravi 12.07.1932. 1 f, 08.07.1933. 5 m, 09.07.1933. 2 m, 10.07.1933. 1 m, 12.07.1933.

$1 \mathrm{~m}$, all leg. Lorković coll. Lorković

Jelenje 13.06.1932. leg. Z. Lorković coll. Lorković $1 \mathrm{~m}$

Posavlje (Ljubljana) 25.06.1955. leg. Z. Lorković coll. Lorković $1 \mathrm{f}$

17. Zygaena exulans (Hohenwarth, 1792)

Zygaena exulans apfelbecki Rebel, 1910

MNE: Suvi Klek (Mt. Durmitor) 15.07.1926. ex coll. Gušić 24 (m+f)

\section{Zygaena osterodensis Reiss, 1921}

Zygaena osterodensis curvata Burgeff, 1926

HR: Ruševo 09.06.1897. ex coll. Koča $1 \mathrm{f}$

Zygaena osterodensis occurs in three different subspecies in Croatia, Z. o. curvata Burgeff, 1926, Z. o. ladina Holik, 1944 and Z. o. koricnensis Reiss, 1922. In Croatia Z. osterodensis was collected on Dilj planina near Ruševo village in Slavonia (KočA, 1901), Josipdol (Mann, 1867) and Planik, Ćićarija Mt (Rebel, 1924). The specimen from Ruševo in the Croatian Natural History Museum derives from same material as the date is the same as from Dilj in KočA (1901) (Fig. 1). This population belongs to Z. o. curvata that is distributed in eastern Austria, Hungary, eastern Slovenia and Slavonia in Croatia. STAuder (1921) also mentiones Z. osterodensis from Istria and Dalmatia, but in his later paper (STAUder, 1930-1932) he does not mention this species any more from Dalmatia. Moreover, exact localities and source from STAUDER (1921) are not known and no specimens have been located in collections. The specimen From Planik (Rebel, 1924) is deposited in the collection of the Naturhistorisches Museum Wien, Austria, with the label - Planik, Istria, 6. July 1913, collected by Rudolf Kitschelt. This female specimen belongs to Z. $o$. ladina, a population group that is distributed in the south-eastern Alps and in western Slovenia including Istria. The population from Josipdol should belong to the Balkan subspecies Z. o. koricnensis but this cannot be proved at the moment as Mann's original material on what his record is based has not been traced in collections so far. However, 
the northernmost known locality of this subspecies in Bosnia and Herzegovina is Drvar and this locality is only $15 \mathrm{~km}$ away from the Croatian border. We can therefore assume that Z. o. koricnensis also occurs in Croatia. Z. o. koricnensis is known from the Dinarid mountains starting from northern Bosnia and Herzegovina (or Croatia if Josipdol can be confirmed) southwards to Baba Mt. and Galičica Planina in Macedonia, but also in southern and eastern Serbia and Bulgaria. We have no records from Z. osterodensis from Croatia for about 100 years. Although, this species is very local and rare in the surrounding countries, it should not be considered as extinct from Croatia but it has to be reconfirmed by newer field research.

\section{Zygaena viciae ([Denis \& Schiffermüller], 1775)}

Zygaena viciae bosniensis Reiss, 1922

HR: Boljara (=Boljare) (Žumberak Mt.) 28.06.1994. leg. M. Vajdić $1 \mathrm{~m} 1 \mathrm{f}$ Hrvatsko-Kupa 27-30.07.1978. leg. L. Mladinov coll. Central $1 \mathrm{~m}$

Sveto Brdo 28.07.1922. ex coll. Gušić $1 \mathrm{~m}$

Vinkovci 03.07.1909. ex. coll. Koča $1 \mathrm{~m}$

SLO: Osilnica 30.06.1971. leg. Mladinov coll. Central 1 f [524] (Mladinov, 1976)

Videm 16.07.1967. leg. Z. Lorković coll. Lorković $1 \mathrm{f}$

\section{Zygaena ephialtes (Linnaeus, 1767)}

Zygaena ephialtes pannonica Holik, 1937

5-spotted ephialtoid yellow

HR: Bosiljevo ex. coll. Kozulić $1 \mathrm{f}$

Dubravkin (=Sofijin) put 12.06.1923. ex. coll. Gušić $1 \mathrm{~m}$

Ivanščica 08.1962. leg. Igalffy coll. Central $1 \mathrm{f}$

Kapela (Lička Plješevica) 26.07.1976. leg. K. Igalffy 1 m 5+1 spotted (Kučınić et al., 1994)

Kutjevo 28.07.1916. ex. coll. Gušić $1 \mathrm{f}$

Lička Plješevica 04.08.1976. leg. K. Igalffy 1 m 1 f (Kučınıć et al., 1994)

Nova Gradiška 29.07.1980. leg. S. Beslač $1 \mathrm{~m}$

Osijek 03.07.1915. ex. coll. Gušić $1 \mathrm{f}$

Pleternica 05.08.1908. ex. coll. Gušić $1 \mathrm{~m}$

Plitvički Ljeskovac 05.07.1920. ex. coll. Gušić 2 m

Senj 19.06.1973. $1 \mathrm{~m}$

Stražnik - Samobor 18.07.1966. leg. Z. Lorković coll. Lorković $1 \mathrm{~m}$

Trnovec 07.1929. 2 m, 03.07.1971. 1 m 1 f, 02.07.1981. 1 f, all leg. Igalffy coll. Central

\section{Zygaena ephialtes corcyrica Rauch, 1981}

5-spotted red ephialtoid

HR: Unije 28.06.1963. leg. L. Mladinov coll. Central 1 m 1 f (Mladinov, 1967)

Rab 08.07.1962. leg. K. Igalffy $2 \mathrm{f}$

$\mathrm{Rab}$ (Rab) 05.07.1964. leg. Z. Lorković coll. Lorković $1 \mathrm{f}$ (in copula with Z. filipendulae)

\section{Zygaena transalpina (Esper, 1780)}

Zygaena transalpina hilfi Reiss, 1922

HR: Bjelolasica (Velika Kapela) 1500 m 23.07.1954. leg. Z. Lorković 1 m

Brod na Kupi 28.06.1971. leg. L. Mladinov coll. Central $1 \mathrm{~m}$ 
Čučak 23.07.1972. leg. L. Mladinov coll. Central $1 \mathrm{f}$ [580]

Duboka 04.07.1955. leg. Z. Lorković coll. Lorković 11 m 8 f

Gračani 06.07.1917. ex. coll. Gušić 1 f (MLadinov, 1958)

Hrvatsko 02.07.1971. 2 m, 24-26.07.1971. 1 f, 01-07.07.1973. 1 f [844], 1 m, all 22.06.1975.

leg. L. Mladinov coll. Central (Mladinov, 1976)

Hrvatsko-Kupa 14.06.1981. leg. L. Mladinov coll. Central 1 f [864]

Kaldanija 25.06.1981. leg. Z. Lorković coll. Lorković $1 \mathrm{f}$

Lokve 03.07.1916. ex coll. Gušić $1 \mathrm{~m}$

Lička Plješevica 04.08.1976. leg. K. Igalffy $1 \mathrm{~m}$

Lipovečka Gradina (Samoborsko gorje) 180 m 22.06.1954. leg. Z. Lorković coll Lorković $1 \mathrm{f}$

Medvedgrad (Medvednica) 27.06.1916. ex. coll. Gušić 1 m [1098] (MLAdinov, 1958)

Podsused, Dolje (Zagreb) larva 28.05.1966. imago 11.06.1966. leg. Z. Lorković coll. Lorković $1 \mathrm{~m}$

Platak (Risnjak) 11.07.1985. leg. Z. Lorković coll. Lorković $1 \mathrm{~m}$

Plitvički Ljeskovac 22.07.1962. leg. Z. Lorković 2 [1491, 1492]

Samobor 19.06.1955. leg. Z. Lorković coll. Lorković $1 \mathrm{~m}$

Zagreb 20.06.1916. ex. coll. Gušić $1 \mathrm{~m}$

SLO: Prisojnik (Julian Alps) 1800-1900 m 14.08.1953. leg. Z. Lorković coll. Lorković 1 m Osilnica 29.06.1971. leg. L. Mladinov coll. Central 4 m [526, 527] (MLAdinov, 1976)

Vršič (north) (Julian Alps) 1250-1400 m 28.07.1954. leg. Z. Lorković coll. Lorković $1 \mathrm{~m}$

\section{Zygaena angelicae Ochsenheimer, 1808}

Zygaena angelicae herzegowinensis Reiss, 1922

BIH: Troglav (Dinara Mt.) Čotrna voda 1320 m 18.07.1962. leg. Z. Lorković coll. Lorković $1 \mathrm{f}$

Sustavac (Treskavica Mt.) 04.07.1968. leg. K. Igalffy coll. Central 2 m [376]

HR: Hrvatsko 26.06.1970. leg. L. Mladinov 1 m [452]

Klek 1100 m 19.07.1953. leg. Z. Lorković coll. Lorković $1 \mathrm{~m} 1 \mathrm{f}$

Klek 17.06.1925. leg. Z. Lorković coll. Lorković 1 m [1085]

Lička Plješevica 04.08.1976. leg. K. Igalffy 3 m 1 f

Mala Bubinka (Lička Plješevica) 08.08.1974. leg. K. Igalffy $1 \mathrm{~m} 1 \mathrm{f}$

Mala Bubinka (Lička Plješevica) 10.08.1974. leg. K. Igalffy $1 \mathrm{f}$

Trnovec 08.1914. leg I. Igalffy $1 \mathrm{~m}$

Vinkovci 15.06.1909. leg. B. Gušić [1086]

Zygaena transalpina hilfi Reiss, 1922 /Zygaena angelicae herzegowinensis Reiss, 1922 hybrids

HR: Čepić, VL 03, 16.06.1999. leg. N. Tvrtković $1 \mathrm{~m}$

Duboka 04.07.1955. leg. Z. Lorković $3 \mathrm{~m}$

Trnovec 08.1914. leg. I. Igalffy $1 \mathrm{~m}$

SLO: Osilnica 29.06.1971. leg. Mladinov $1 \mathrm{~m} 1 \mathrm{f}$ [528]

23. Zygaena filipendulae (Linnaeus, 1758)

Zygaena filipendulae illyrica Holik. 1943

BIH: Sustavac (Treskavica Mt.) 04.07.1968. leg. K. Igalffy coll Central 1 m [373] 
HR: Klek 1100 m 19.07.1953. leg. Z. Lorković coll. Lorković 1 f

Klek 14.07.1916. ex. coll. Gušić 1 m

Krpelj 13.07.1916. ex. coll. Gušić 1 f

Leska (Risnjak) 24.06.1994. leg. M. Šašić 2 m

Lokve 05.08.1955. leg. Lorković coll. Lorković $1 \mathrm{f}$

Mala Bubinka (Lička Plješevica) 10.08.1974. leg. K. Igalffy 1 f (Kučınıć et al., 1994)

Mala Kapela 06.1960. leg. Igalffy 2 m (Kučinıć et al., 1994)

Ogulin 13.07.1916. ex. coll. Gušić $1 \mathrm{~m}$

MNE: Lovćen 27.07.1926. ex. coll. Gušić $1 \mathrm{f}$

Žabljak (Mt. Durmitor) 24.07.1926. ex. coll. Gušić $1 \mathrm{~m}$

MK: Lisec (Šar-planina Mt.) 1100 m 29.07.1952. leg. Z. Lorković coll. Lorković $1 \mathrm{~m}$

Zygaena filipendulae polygalae (Esper, 1783)

HR: Bosiljevo ex. coll. Kozulić 1 f 1 m (Mladinov, 1976)

Bregana 10.06.1987. leg. Z. Lorković coll. Lorković $1 \mathrm{~m}$

Brod na Kupi 28.06.1971. leg. L. Mladinov coll. Central $1 \mathrm{~m} 1 \mathrm{f}$

Buk (Psunj Mt.) 24.06.1986. leg. F. Perović $1 \mathrm{~m}$

Cmrok (Zagreb) ex. coll. Gušić 13.07.1917. $1 \mathrm{f}$

Duboka (Papuk Mt.) 04.07.1955. leg. Z. Lorković coll. Lorković $1 \mathrm{~m} 1 \mathrm{f}$

Gornje Čučerje (Zagreb) 01.07.1990. leg. Z. Lorković coll. Lorković $1 \mathrm{~m}$

Hrvatsko 26.06.1970. 1 m [454], 02. 07.1971. 1 m [529], 15.06.1972. 1 m [579], 1-7.07.1973.

$1 \mathrm{~m}$ [846], 22.06.1975. pupa, 03.07.1975. imago $1 \mathrm{~m}$, all leg. L. Mladinov coll. Central (MLadinov, 1976)

Hrvatsko - Kupa 10.06.1979. 4 m [843], 14.06.1981. 1 m 2 f [861] leg. L. Mladinov coll. Central

Lipovečka Gradina (Samoborsko Gorje) 180 m 22.06.1954. leg. Z. Lorković coll. Lorković $2 \mathrm{~m}$

Macelj 08.08.1948. leg. I. Igalffy coll Central $1 \mathrm{~m}$

Maksimir (Zagreb) 11.07.1924. 1 m 09.07.1931. $1 \mathrm{~m}, 19.07 .1932 .1 \mathrm{~m}$, all ex. coll. Valjavec (Mladinov, 1958)

Nova Gradiška 21.07.1980. 1 m, 25.07.1980. 1 m, 29.07.1980. 1 m, 01.08.1980. 1 m, 08.08.1980. 1 f, leg. S. Beslač

Palačnik 19.06.1955. leg. Z. Lorković coll. Lorković 4 m 4 f (Mladinov, 1958)

Podsused 09.07.1923. ex. Coll. Gušić 1 m [845]

Severin 28.06.1972. leg. Z. Lorković coll. Lorković $1 \mathrm{~m}$

Slatina 08.08.1915. ex. Coll. Gušić $1 \mathrm{f}$

Sveta Gera 19.06.1917. ex. Coll. Gušić 1 m

Šestine - Kraljevec 22.07.1923. ex. Coll. Valjavec $1 \mathrm{f}$ (MLAdinov, 1958)

Trnovec 06.1914. 1 m, 07.1929. 2 m 2 f, 16.07.1981. 1 f, all leg. Igalffy (Mladinov, 1976)

Turopolje, Drnek 15.06.1993. leg. F. Perović $1 \mathrm{~m}$

Vinkovci 04.07.1909. ex. Coll. Koča $1 \mathrm{f}$

SLO: Ložec - Kupa 23.06.1974. 1 f, 21.06.1976. 1m both leg. L. Mladinov coll. Central Ložec 13.07.1980. leg. L. Mladinov coll. Central 3 m 2 f [863, 862]

Osilnica 26.06.1970. $1 \mathrm{~m}$ [451] (MLAdinov, 1976)

Osilnica 20.06.1972. leg. L. Mladinov coll. Central $1 \mathrm{~m}$ (Mladinov, 1976)

Ribjek 13.07.1980. leg. L. Mladinov coll. Central $2 \mathrm{~m}$ 
Srobotnik 14.06.1981. leg. L. Mladinov coll. Central $1 \mathrm{~m}$

Videm 16.07.1967. leg. Z. Lorković coll. Lorković $3 \mathrm{~m} 1 \mathrm{f}$

SRB: Remeta (Fruška Gora Mt.) 16.07.1975. leg. Z. Lorković coll. Lorković $1 \mathrm{~m}$

\section{Zygaena filipendulae zarana Burgeff, 1926}

HR: Barbati (Pag) 25.06.1956. $1 \mathrm{~m}$ [84]

Brseč 05.06.1970. 2 m 2 f, 06.06.1970.1 m, all leg. K. Igalffy

Brseč, Golovik 06.1973. leg. K. Igalffy $2 \mathrm{~m} 2 \mathrm{f}$

Caska (Pag) 23.06.1956. 1 m, 08.06.1958. $1 \mathrm{~m} 1 \mathrm{f}$ [82], 09.06.1958. $1 \mathrm{~m}$ [83],10.06.1958. $1 \mathrm{~m}$, 21.06.1960. $1 \mathrm{~m}, 22.06 .1960 .1 \mathrm{f}[231], 24.06 .1960 .1 \mathrm{f}, 25.06 .1962 .2 \mathrm{~m}$ [230], 26.06.1960. $1 \mathrm{f}$ [169], 16.08.1960. 3 m 1 f [166, 168, 167, 165] all leg. L. Mladinov coll. Central (MLAdinov, 1965)

Čedanj 13.07.1980. leg. L. Mladinov coll. Central $1 \mathrm{f}$

Jasenak (Velika Kapela) 650 m 22.07.1954. leg. Lorković coll. Lorković 1 m [1091] $1 \mathrm{f}$ Jelenje-Grobnik (15 km NW Rijeka, Grobničke Alpe) 700 m 09.07.1985. leg. Z. Lorković $1 \mathrm{~m}$

Kolansko blato (Pag) 11.06.1958. leg. Mladinov coll. Central 3 m (Mladinov, 1965)

Male Srakane 18.06.1962. leg. A. Magerle coll. Central $1 \mathrm{~m}$

Mali Lošinj 23.05.1974. leg. K. Igalffy 2 m, 2 f

Mošćenička Draga 14.09.1965. coll. Lorković 2 m (both 6-spotted melanistic) $3 \mathrm{f}$

Novalja (Pag) 26.07.1996. leg. G. Gjerapić $1 \mathrm{~m}$

Novi Vinodolski 08.1946. $1 \mathrm{~m}, 15.06 .1975 .1 \mathrm{~m}$ both leg. Z. Lorković coll. Lorković

Rab 08.07.1962. leg. K. Igalffy $2 \mathrm{~m}$

$\mathrm{Rab}(\mathrm{Rab})$ 05.07.1964. leg. Z. Lorković $1 \mathrm{~m}$ (in copula with Z. ephialtes)

Rovinj 05.10.1955. 1 f, 05.06.1960. 1 m, both leg. Z. Lorković coll. Lorković

Senj 19.06.1973. 2 f leg. Mladinov

Stara Novalja (Pag) 13.06.1958. leg. Mladinov coll. Central 2 m (Mladinov, 1965)

Susak 13.09.1961. $1 \mathrm{~m}, 06.1962 .1 \mathrm{~m}, 29.08 .1963 .1 \mathrm{~m}$ all coll. Central

Trnovec 06.1914. $1 \mathrm{~m}, 07.1929 .2 \mathrm{~m} 2 \mathrm{f}, 16.07 .1981 .1 \mathrm{f}$, all leg. Igalffy

Vozilići 30.08.1973. 1 m 1 f, 29.08.1975. 1 m, 05.09.1975. 2 m, 22.09.1976. 1 m, 29.06.1978.

$1 \mathrm{~m}, 27.09 .1978 .1 \mathrm{f}$, all leg. F. Perović

Unije 26.06.1963. leg. L. Mladinov coll. Central $1 \mathrm{~m}$ (MLAdinov, 1967)

Unije 02.09.1963. leg. L. Mladinov coll. Central 1 m 1 f (MLadinov, 1967)

Zadar 1887. ex. Coll. Locke $1 \mathrm{~m}$

\section{Zygaena lonicerae (Scheven, 1777)}

Zygaena lonicerae lonicerae (Scheven, 1777)

HR: Bosiljevo ex. Coll. Kozulić 1 f (Mladinov, 1976)

Crni vrh (Plitvice) 1000 m 26.07.1961. leg. Z. Lorković coll. Lorković $1 \mathrm{~m}$

Fužine, Lič 800 m 05.07.1952. leg. Z. Lorković coll. Lorković 1f

Grdanjci (Bregana) 21.06.1942. leg. Z. Lorković coll. Lorković 1 f

Hrvatsko 02.07.1971. leg. L. Mladinov coll. Central 1 m [531] (Mladinov, 1976)

Hrvatsko 01-07.07.1973. leg. L. Mladinov coll. Central 2 m 1 f (Mladinov, 1976)

Hrvatsko 26.06.1970. leg. L. Mladinov coll. Central 2 f [453, 455] (MLAdinov, 1976)

Lokve 03.08.1916. ex. Coll. Gušić $1 \mathrm{f}$

Lipa (Zagreb) 08.07.1917. ex. Coll. Gušić $1 \mathrm{f}$ 
Ložec - Kupa 21.06.1975. leg. L. Mladinov coll. Central 1 f (MLadinov, 1976)

Lička Plješevica 04.08.1976. leg. K. Igalffy $1 \mathrm{f}$

Mali Lošinj 21.05.1974. leg. K. Igalffy $1 \mathrm{f}$

Oštarije pass (Mt. Velebit) 820 m 27.07.1983. leg. Z. Lorković coll. Lorković $1 \mathrm{f}$

Plitvički Leskovac 02.07.1951. leg. Z. Lorković coll. Lorković $1 \mathrm{f}$

Vinkovci 03.07.1908. ex. Coll. Koča $1 \mathrm{f}$

SLO: Osilnica 26.07.1970. coll. Central 2 f [453, 455] (MLAdinov, 1976)

Osilnica 29.06.1971. leg. L. Mladinov coll. Central 2 m 1 f [533, 530, 532] (Mladinov, 1976)

Pišnica (Julian Alps) 900 m 28.07.1954. leg. Lorković coll. Lorković $1 \mathrm{f}$

Pokojišče 25.05.1957. leg. Z. Lorković coll. Lorković $1 \mathrm{~m}$

Škocjanske jame, Matavun 07.06.1981. leg. Z. Lorković coll. Lorković $1 \mathrm{f}$

\section{CONCLUSION}

Almost all species occurring in Croatia are represented in the collections of the Croatian Natural History Museum. Despite the small number of specimens stored in this museum Zygaenidae of Croatia are well studied if compared with other countries of the Balkan Peninsula. As the majority of papers deal with the coastal area of Croatia (Dalmatia), this collection is valuable since there are specimens from other areas. The comparison of new field observations with historical data allows us to comment on changes in the distribution of species. These changes can have their origin in a natural shift of species areal, lack of field work (not sufficient studies undertaken) or in events like described above (true destruction events in habitats).

Received May 9, 2016

\section{REFERENCES}

Abafi-Aigner L., Pavel J. \& F. Uhryk 1896: Fauna regni Hungariae/ Lepidoptera. 1-78.

Abafi-Aigner, L., 1910: Adaléka Magyar Tengermellék, Horvátország és Dalmáczia lepkefaunájához. Rovartani Lapok 17, 55-57, 71-105.

Bartol, B., Bartol, V., \& Š. Michieli, (1964): Beitrag zur Kenntnis der Makrolepidopterenfauna der adriatischen Insel Krk. Nachrichtenblatt der Bayerischen Entomologen 13(4): 33-36, 44-48, 55-59.

Вонатsсн, O., 1892: Beitrage zur Lepidopteren-Fauna Slavoniens. Wiener Entomologischen Vereines 2, 31-50.

Carnelutti, J., 1994: Modernisiertes „Verzeichnis der bei Knin gesammelten Schmetterlinge“ von I. Hafner. Natura Croatica 3, 185-223.

Carrara, F., 1846: La Dalmazia descritta. Fratelli Battara tipografi editori, pp. 130, Zara(=Zadar). [in Italian]

Duponchel, M. P. A. J., 1844[1846]: Catalogue méthodique des lépidoptères d’Europe. Méquignon-Marvis, pp. 523, Paris.

Efetov, K. A. \& G. M. Tarmann, 1999: Forester Moths: The genera Theresimima Strand, 1917, Rhagades Wallengren, 1863, Jordanita Verity, 1946, and Adscita Retzius, 1783 (Lepidoptera: Zygaenidae, Procridinae) 192 pp., figs 1-415, 12 col. pls. Stenstrup.

GalvaGni, E., 1902: Beiträge zur Kenntis der Fauna einiger dalmatinischer Inseln. Verhandlungen der zoologisch-botanischen Gesellschaft in Wien 362-388.

Habeler, H., 2003: Die Schmetterlinge der Adria-Insel Krk. Buchreihe zur entomologie Esperiana, Graz, $221 \mathrm{pp}$.

Hafner, I., 1994: Verzeichnis der bei Knin gesammelten Schmetterlinge (Lepidoptera). Natura Croatica 3, 119-184. 
Hofmann, A. \& W. G., Tremewan, 1996: A Systematic Catalogue of the Zygaeninae (Lepidoptera: Zygaenidae), Colchester, $251 \mathrm{pp}$.

JAKšıć, P. \& G., Ristić, 1999: New and rare species of Lepidoptera in Yugoslavia. Acta entomologica Serbica, 4(1-2), 63-74.

KočA, GJ., 1900: Prilog fauni gore Papuka i njegove okoline. Glasnik Hrvatskog naravoslovnog društva 12, 1-35.

KRANJČEV, R., 1985: Odnos faune makrolepidoptera prema prirodnim i antropogenim staništima Podravine i podravskih pijesaka (I). Podravski zbornik 11, 200-226

Kučinić, M., IgAllfy, K., ŠAšić, M. \& S. BALEN, 1994: A contribution on the Heterocera Fauna (Insecta, Lepidoptera) of the Central-mountain part (Risnjak \& Lička Plješevica) of the Republic of Croatia. Natura Croatica 3(1), 23-40.

LEDERER, J., 1853: Versuch, die europäischen Lepidopteren (einschliessig der ihrem Habitus nach noch zur europäischen Fauna gehörigen Arten Labradors, der asiatischen Türkei und des asiatischen Russlands) in möglichst natürliche Reihenfolge zu stellen, nebst Bemerkungen zu einigen Familien und Arten. II. Abteilung: Die Heteroceren. Verhandlungen (Abhandlungen) des Zoologisch-botanischen Vereins in Wien 2, 65-126.

ManN, J., 1857: Verzeichniss der im Jahre 1853 in der Gegend von Fiume gesammelten Schmetterlinge. Wiener Entomologische Monatschrift. Wien 1, 139-159, 161-189.

ManN, J., 1867: Schmetterlinge gesammelt im J. 1866 um Josefsthal in der croat. Militärgrenze Verhandlungen der kaiserlich-königlichen zoologisch-botanischen Gesellschaft in Wien, XVII, 63-76.

Mann J., 1869: Lepidoptern gesammelt während dreier Reisen nach Dalmatien in den Jahren 1850, 1862 und 1868. Verhandlungen der zoologisch-botanischen Gesellschaft in Wien 19, 373-388.

Mladinov, L., 1958: Popis noćnih leptira (Noctua) Zagreba i okolice. Hrvatski narodni zoološki muzej, Entomološki odjel. 1, 1-61.

Mladinov, L., 1965: Rezultati istraživanja faune Rhopalocera i Heterocera otoka Paga. Biološki glasnik $18,37-48$.

Mladinov, L., 1967: Fauna leptira jadranskog otoka Unije. Biološki glasnik 20, 151-164.

Mladinov, L., 1976: Lepidoptera iz doline gornjeg toka rijeke Kupe II. Bombyces i Sphinges. Acta entomologica Jugoslavica 12, 89-98.

MLadinov L., 1978: Prvi dodatak poznavanju faune Macrolepidoptera iz doline gornjeg toka rijeke Kupe, Acta entomologica Jugoslavica, 14, 63-67.

Mоuсна, J., 1966: Zur Kenntnis der Schmetterlingsfauna Jugoslawiens (Lepidoptera). Entomologische Nachrichten 10, 49-53.

NAhirnić, A., TARMAnN, G. M. \& P. JaKšIĆ, 2012: Zygaenidae (Lepidoptera) in the collections of the Natural History Museum in Belgrade (Serbia). Bulletin of the Natural History Museum, 5, 73-94.

NAhirnić, A., TARMAnN G. M. \& P. JAKŠIĆ, 2013: New data on rare Zygaenidae from the Balkan Peninsula. XVIII Europaean Congress of Lepidopterology, 29 July - 4 August 2013, Blagoevgrad, Bulgaria, p. 58.

RAUch, H., 1981: Ein Beitrag zur Zygaenenfauna der Insel Korčula in Dalmatien (Jugoslawien), (Lepidoptera, Zygaenidae) Würzburg, 64-71

ReвеL, H., 1910: Fr. Berge's Schmetterlingsbuch nach dem gegenqwärtigen Stand der Lepidopterologie. (Neunte Auflage). E. Schweizerbart'sche Verlagsbuchhandlung. Nägele \& Dr. Sproesser, pp. I-VI+1$114+1-509,53$ pls. Stuttgart.

Rebel, H., 1911-1913: Lepidopteren aus dem Gebiete des Monte Maggiore in Istrien. Wiener entomologischen Vereines, XXI, 97-110; XXII, 227-240; XXIII, 177-205.

RebeL, H., 1924: Lepidopteren aus dem Gebiete des Monte Maggiore in Istrien. III. Nachtrag. Jahresbericht des Wiener Entomologischen Vereines 30, 25-35.

SchawERDA, K., 1921: Beiträge zur Lepidopterenfauna der kroatischen Küste und Neubeschreibungen. Deutche Entomologische Zeitschrift Iris 35, 111-138.

Schwingenschuss, L. \& F. WAgner, 1925-1927: Beitrag zur Macrolepidopteren Fauna Suddalmatiens insbesondere der Umgebung von Gravosa. Zeitschrift des Österreichischen Entomologen-Vereines, X, 53-57, 66-69, 78-82, 116-119; XI, 1-3, 9-13, 26-29, 53-54, 67-72, 74-70, 81-86; XII, 45-50, 62-64, 68-72, 73-75.

StAuder, H., 1921: Die Zygaenidenarmut der Adriatischen Inseln. Societas entomologica 36, 1-4, 6-7. 
STAUDER, H., 1930-1932: Die Schmetterlingsfauna der illyro-adriatischen Festland- und Inselzone (Faunula Illyro Adriatica).- Entomologischer Anzeiger Wien 10, 203-204, 249-252, 275-276, 294-297, 309-310, 351-352, 374-377, 436-437; 12, 35-39, 53.

TARmann, G. M., 2000a: Agriculture and Zygaenidae in Alpine valleys - a case study. Abstracts of the VII. International Symposium on Zygaenidae, Innsbruck 4-8 September 2000, 31.

TARmann, G. M., 2000b: Zygaenidae und Spritzmitteleinsatz im oberen Vinschgau. Zoologische und botanische Forschungen in Südtirol 29.9.-1.10.2000 in Bozen, 37-38.

Tarmann, G. M., 2004: Zygaenid Moths of Australia. A revision of the Australian Zygaenidae (Procridinae: Artonini). 352 pp., 64 colour plates, 448 figs. CSIRO Publishing, Collingwood.

TARMAnN, G. M., 2009: Die Vinschger Trockenrasen - ein Zustandsbericht auf Basis der Bioindikatoren Tagfalter und Widderchen (Lepidoptera: Rhopalocera, Zygaenidae). Wissenschaftliches Jahrbuch der Tiroler Landesmuseen 2, 306-350, figs 1-42, 1 map.

Vuкотіnović, LJ., 1879: Fauna leptirah u okolišu Zagrebačkom. Rad JAZU 48: 1-129. 